\title{
Study on the Brain Stereotaxic Method of Carp Aquatic Animal-robot
}

\author{
Yong $\mathrm{PENG}^{1 *}$, Cong-shan $\mathrm{GUO}^{1}$, Yang-yang $\mathrm{SU}^{1}$, Zhan-qiu \\ WANG $^{2}$, Pei- hua SU ${ }^{1}$, Dan DU², Wei-chao SHEN ${ }^{1}$, Ya-kun JU' ${ }^{1}, R u$ \\ TIAN ${ }^{1}$, Xiang-qian ZHOU ${ }^{1}$, Fan $\mathrm{ZHAN}^{1}$, Jie $\mathrm{LI}^{1}$ and $\mathrm{Li}^{-j u a n} \mathrm{YING}^{1}$ \\ ${ }^{1}$ Department of Biomedical Engineering, College of Electrical Engineering, Yanshan \\ University, Qinhuangdao 066004, China \\ ${ }^{2}$ Department of Magnetic Resonance, The First Hospital of Qinhuangdao Town, \\ Qinhuangdao 066000, China \\ ${ }^{*}$ Corresponding author, e-mail: PY81@sina.com
}

Keywords: Carp, Aquatic animal-robot, CT imaging, Skull partition, Brain stereotaxic positioning, Biological control.

\begin{abstract}
Purposes] Our aim is to explore the brain stereotaxic method of aquatic animal-robots. [Methods] Carps were anesthetized by Eugenol. The carp skulls and brain were observed by the method of anatomy. Carp brain structure and size were obtained by three-dimension images which were structured by CT. The brain stereotaxic method was confirmed by setting up a $3 \mathrm{~d}$ coordinate system of brain and dividing carp skull surface into 8 location areas. The $3 \mathrm{~d}$ coordinate system of brain was built according to the principle of brain stereotaxic coordinates, the bony marks and the characteristics of carp skull. And the 8 location areas were used for implanting electrodes. Stimulating electrodes were implanted into cerebral motor areas by the application of brain stereotaxic method. [Results] The carp-robot went forward and turned successfully by controlling in underwater experiment. [Conclusion] The results showed that the brain stereotaxic method is feasible and reliable for carp-robot.
\end{abstract}

\section{Introduction}

Fish has had flexible movement capacity in water. Aquatic-robot can not only use its own flexible motor, but also work in accordance with the wishes of human. So aquatic-robot has broad prospect for the development in ocean expedition, marine environmental monitoring, searching and rescuing underwater. Surveying the quality of tunnels and dam, prediction of earthquake and tsunami etc[1]. Stereotaxic technology is needed to orient accurately on brain orient injection, stimulation, damage, EEG extraction and biological behavior control etc [2]. To achieve behavior control of fish, the brain cerebral nucleis should be known. Therefore, establishing brain stereotaxic method is the basis for the research of aquatic-robot.

In 1893, the theory of stereotaxic technology was introduced and a positioning equipment was designed by Dittmen. Then the wizard probes were applied in nerve physiology at the first time. In 1908, the first three-dimensional stereotactic instrument was developed by English scientists Robert Henry Clarke and Victory Horsley. The instrument was designed by adopting orthogonal three-dimensional plane coordinate system which was established on the basis of the experimental animal skull marks and the characteristics of brain anatomical structure [3]. On the basis of the principle of the stereotactic instrument, some auxiliary means were adopted to locate accurately in most subsequently developed stereotactic instruments. Such as the 
combination of stereotaxic instruments with Magnetic Resonance Imaging (MRI), Digital Subtraction Angiography (DSA), Positron Emission Computed Tomography (PET) etc. Accurate positioning of any target and exact measurement of the volume were achieved in brain.

In this study, the brain stereotaxic method was based on the positioning principle of Robert Henry Clarke's three-dimensional plane coordinate system which put the origin of coordinates as the center and established horizontal, sagittal plane and coronal plane. By Computer Tomography (CT) technology, digital brain stereotaxic instrument, carp skull crossed partition, three-dimensional positioning was carried on carp brain, and brain stereotaxic method was built. The brain stereotaxic method is suitable for teleosts such as carps and it contributes to reliable fixation and accurate positioning for teleost brain and the method provides guarantees for carp brain function research.

\section{Materials and Equipments}

\section{Experimental Animal}

50 adult carps, at the average weight of $1.05 \pm 0.11 \mathrm{~kg}$, and at the average length of $38.20 \pm 1.26 \mathrm{~cm}$, were bought from the market of Qinhuangdao aquatic product.

\section{Experimental Reagents and Equipments}

Eugenol (Shanghai Medical Instruments Co., Ltd. Dental Material Factory,China, Batch number 201002).

Computer Tomography (General Electric Company,America,product model Discovery CT 590RT).Micro hand drill (Cixi Baohe instrument Co., Ltd. China, product model P-600).Digital display brain stereotaxic instrument (Stoeliting company, American, product model 620 WHEAT LANE). DC stabilized power supply (Xuzhou electronic instrument factory,china,product model PD1723B). PXI virtual instrument platform (National Instruments Co.,Ltd. American). Self-made electrodes.

\section{Experimental Method}

\section{Carp Anesthesia}

The carps $(\mathrm{n}=50)$ were put into anaesthetic solution with the concentration of $0.36 \mathrm{~g} / \mathrm{L}$.

\section{Carp Brain Dissection Experiment}

Anaesthetic carps were put on the laboratory bench and then dissected. Anatomy marks were observed clearly on the skull surface, such as the boundary-coronal suture which is between parietal bone and frontal bone, the sagittal suture which is between parietal bone and parietal, and between frontal bone and frontal. The anatomy marks are shown in Fig.1. Coronal suture and sagittal suture are important marks in brain stereotaxic method. According to the carp brain external characteristics, these marks which were looked from outside are not obvious, but the intersection of the parietal and occipital are obvious. 


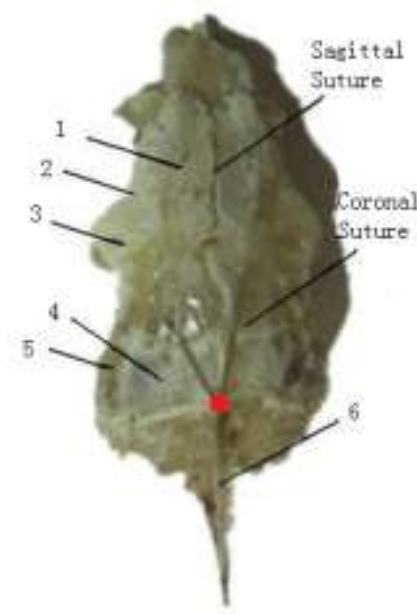

(a) front view

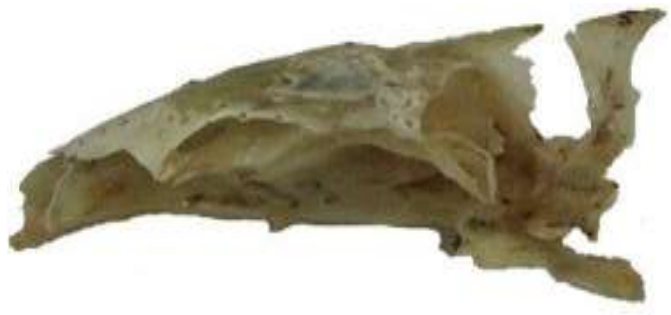

(b)side view

Figure 1. Carp skull anatomy (1. frontal bone, 2, 3 orbital bone, 4. Parietal bone, 5. Operculum, 6. supraoccipital bone)

\section{Brain Imaging Observation of Carp}

Anatomical work can be reduced by CT scanning skull structure and brain tissue of carp [4]. The carp brain CT planar images are shown in Fig.2 and the carp brain CT stereograms are shown in Fig.3. In this study, carp brain with the mode of lateral position and head going first was scanned by CT equipment, and brain cross-sectional anatomy images were obtained. The sizes of brain tissues' boundaries and the distances between brain regions' boundaries and medium imaging were measured by VisionPACS software. The medium imaging was contribute to the data of relationship and distances between extracranial feature points and brain regions.

\section{Establishment of Brain Stereotactic Method of Carp}

Determination of Three-dimensional Planes. According to the bony marks and characteristics of carp skull, the three-dimensional coordinate system was determinated, it is shown in Fig.4. The intersection of the coronal suture and the sagittal suture was determined as the origin $\mathrm{O}$, namely the joint of carp brain and the first piece of fish scales in trunk. The jawbone midpoint was defined as the lip point $B$. The intersection of the extension line of the origin and the vertical which was across the lip point was the reference point. The line which was across the origin and the reference point was defined as the baseline. The plane which was across the baseline and parallelled to the line which was across the lowest points of the orbital edges was defined as the horizontal datum plane. The plane which was across the baseline and was perpendicular to horizontal datumplane was defined as the sagittal datum plane. Then the plane which was across the origin and was perpendicular to horizontal datum plane and the sagittal datum plane was defined as the coronal datum plane.

Establishment of Carp Brain Location Areas. Carp skull was divided into two parts by sagittal suture and the two parts were symmetrical. When the skull was divided, the skin of skull was scraped off. Then a straight line L2 was drew with a sign pen, it is along the sagittal suture from the rhynchodaenm to the origin. The superior orbital edge of left eye was chosen as the benchmark, a straight line L1 which parallelled to L2 was drew. The superior orbital edge of right eye was chosen as the benchmark, a straight line L3 which parallelled to L2 was drew. 
L4 which was perpendicular to L2 was drew, and it was next to the front orbital edges of both eyes. L5 which was perpendicular to L2 and parallelld to L4 was drew, L5 was next to posterior orbital edges of both eyes. The joints of the operculum bones and orbital bones were chosen as reference points, draw L6 which was across the reference points, was perpendicular to L2 and parallelled to L4. The joint of brain and the first piece of fish scales in trunk was chosen as the reference point, L7, which was across reference point,was perpendicular to L2 and parallelled to L4, was drew. Posterior edges of both operculum bones were chosen as reference point, draw L8 which was across reference point, was perpendicular to L2 and parallelled to L4. The specific divisions are shown in Fig.5.The carp skull was divided into 8 location areas, namely A,B,C,D,E,F,G,H.

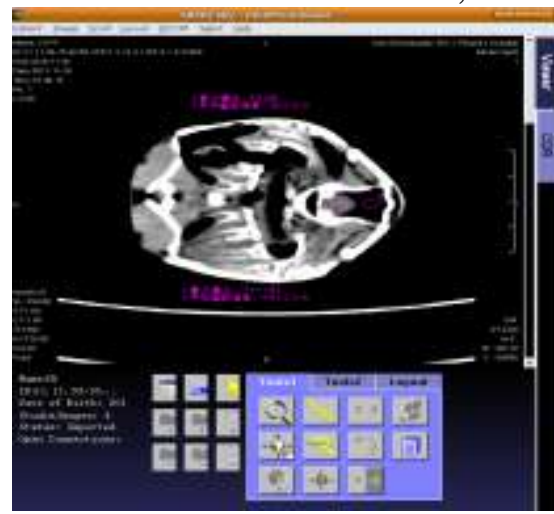

(a) Top view

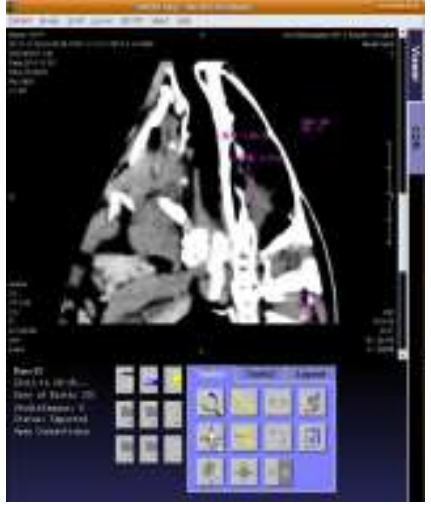

(b) Axial view

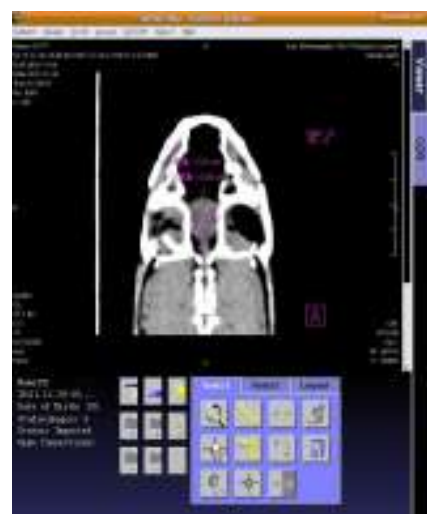

(c) Side view

Figure 2 Carp brain CT planar images

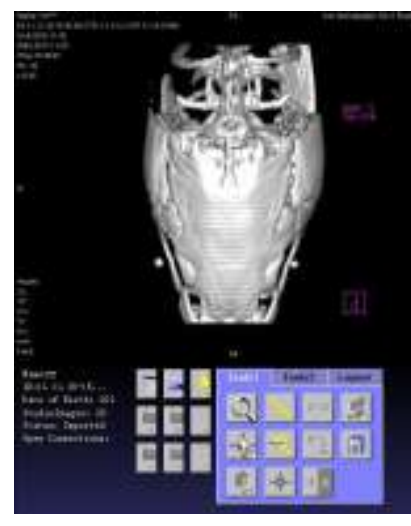

(a) Top view
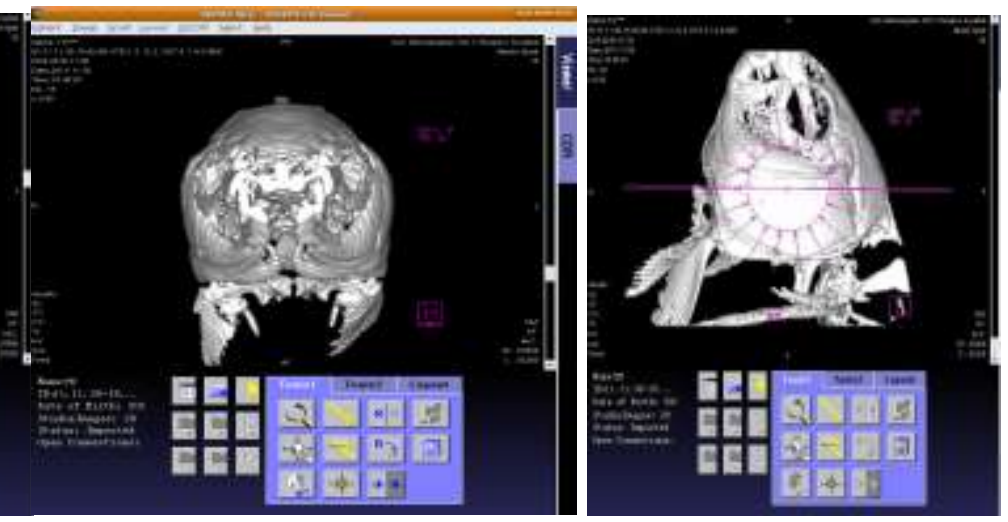

(c) Side view

Figure 3 Carp brain CT stereograms 


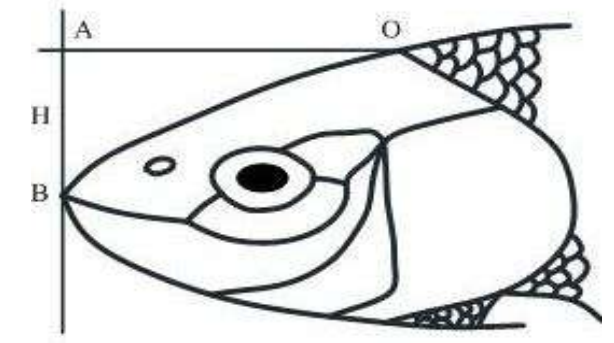

Figure 4. The principle diagram of carp brain

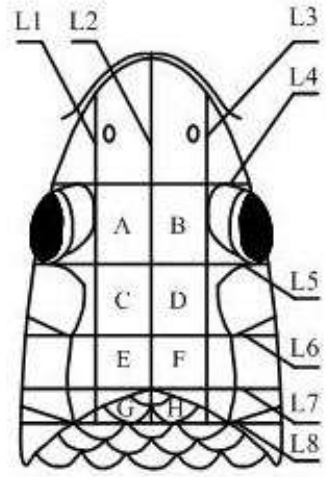

Figure 5. The sketch of divisions on carp skull

stereotaxic coordinate system

Carp Brain Stereotaxic Method. The carp was fixed on brain stereotaxic instrument. The three fiducial planes of skull corresponded to the instrument fiducial planes. The both superior edges of operculum bones were clamped with left and right eye rod holder. The relative distance between left and right eye rod holder was adjusted with vernier scale value of eyebar to keep sagittal datum of skull and relative fiducial plane of the instrument parallel. Upper and lower jaws were opened, and carp rhynchodaenm was clamped with a adapter. The trunk was fixed by self-made clamping devices. The specific image of carp being fixed on brain stereotaxic instrument is shown in Fig.6. X-the left and right sides, parallelled to the intersecting line of the horizontal datum and coronary datum, was from left side to right side.Y-the front and back sides, parallelled to the intersecting line of horizontal datum and sagittal datum, was from tail to rhynchodaenm. Z-depth, was perpendicular to horizontal datum, was from backside to belly.

\section{The Underwater Behavior Control Test of Carp-Robot}

4 electrodes were implanted into the carp's brain, which included 1 reference electrode and 3 stimulating electrodes. Carps were given rectangular pulse signal by virtual stimulator of PXI modular instrument box and virtual stimulating interface. Stimulus parameters were that the voltage was $4.00-8.00 \mathrm{~V}$, the frequency was $1-50 \mathrm{~Hz}$. Every site was repeated 3 times, and every interval was $10 \mathrm{~min}$. The Carp-robot underwater behavior control test is shown in Fig.7.

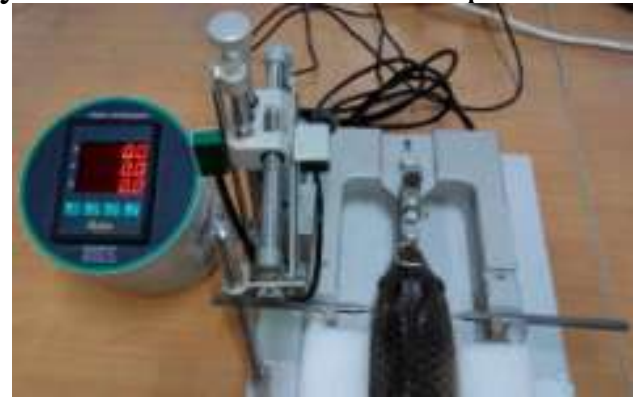

Figure 6. The carp was fixed on brain stereotaxic instrument

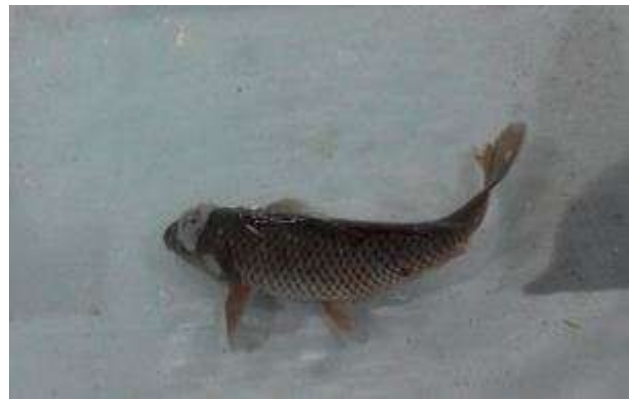

Figure 7. The underwater behavior control test of Carp-robot 


\section{Results}

The images of carp skull structure and brain tissues were obtained by CT. The size of encephalocoele width is $1.83 \pm 0.11 \mathrm{~cm}$, and the distance between cerebrum and medulla oblongata was $2.19 \pm 0.07 \mathrm{~cm}$. According to brain stereotaxis principle of rectangular three-dimensional plane coordinate system, the intersection of coronal suture and sagittal suture was determined as the origin, and then the horizontal, sagittal plane, coronal plane and three-dimensional coordinate system were established. According to the skull bony landmarks, the skull surface was divided into 8 location areas. This method could improve the accuracy of positioning. Stimulating electrodes were implanted into cerebral motor areas which produced corresponding movements by the application of brain stereotaxic method. The carp-robot went forward, turned left and right by controlling successfully in underwater experiment. The success rates of controlling advancing, turning left and right severally were 75\%, 90\%, 85\%.

\section{Discussion}

The carp skull structures and brain tissue structures were known well by CT images and the dissecting experiments. Those provided imaging data for implanting electrodes and foundation for establishing a coordinate system and drawing lines on skull surface. The $3 \mathrm{~d}$ coordinate system which regarded the intersection of the coronal suture and the sagittal suture as the origin was more accurate for the locations of brain tissues , and it provides conditions for implanting electrodes accurately. The carp aquatic animal-robot was controlled effectively, so we think that this method is feasible and reliable.

\section{Conclusions}

This brain stereotaxic method is accurate, simple and convenient for operations, and it may provide a new method for brain stereotaxic positioning of teleosts such as carp.

\section{Acknowledgement}

This project was supported by National Natural Science Foundation of China (project number: 61573305), and $\mathrm{PhD}$ Foundation in Yanshan University in China (project number: B702).

\section{References}

[1] X.W. Guo, The Pentagon want to recruit sharks as spies, China National Defense, 2006.In Chinese.

[2] Z, Liu, Fundamental research of biological behavior control of aquatic animals-robot, D. Yanshan University, Qin Huang Dao, China. 2012.In Chinese.

[3] R.H. Clarke, The linacra lecture on the function of the so called "motor" area of the brain, J. Br Med J. 21 (2009) 125-132.

[4] L.N. Gao, W.G. Chen, The application development and prospect of CT technology, J.CT theory and application research.1 (2009) 99-109.In Chinese. 